\title{
miR-375 inhibits IFN- $\gamma$-induced programmed death 1 ligand 1 surface expression in head and neck squamous cell carcinoma cells by blocking JAK2/STAT1 signaling
}

\author{
QINGWEI WU, YINGYING ZHAO, YIYUAN SUN, XIAOJUN YAN and PEIHUA WANG \\ Department of Ear, Nose and Throat, The Ninth Affliated Hospital of Shanghai Jiao Tong University, \\ Shanghai 200011, P.R. China
}

Received July 4, 2017; Accepted December 12, 2017

DOI: 10.3892/or.2018.6177

\begin{abstract}
Upregulation of programmed death 1 ligand 1 (PD-L1) in cancer cells and its ligation to PD-1 on T cells facilitates cancer cell escape from immune surveillance. Therapies with PD-1 or PD-L1 antibodies have resulted in marked clinical responses in various cancer types. Hence, modulators that inhibit PD-L1 expression in cancer cells may serve as a novel strategy by which to enhance host immune responses. In the present study, we investigated the effects of miR-375 on PD-L1 expression in head and neck squamous cell carcinoma (HNSCC) cells by qRT-PCR and western blot analyses. We confirmed that miR-375 inhibited IFN- $\gamma$-induced PD-L1 surface expression in HNSCC cells, and we observed that Janus kinase 2 (JAK2) is a bona fide target of miR-375 and further activated signal transducer and activator of transcription 1 (STAT1). Additionally, miR-375-mediated inhibition of PD-L1 expression was dependent on the JAK2/STAT1 pathway. Therefore, by attenuating PD-1/PD-L1 signaling, miR-375 may also serve as a modulator to increase the cell immune responses to HNSCC.
\end{abstract}

\section{Introduction}

Immunotherapy has shown excellent promise for various types of cancers (1). The most promising approach to activate or enhance antitumor immunotherapy is the blockade of immune checkpoints. Immune checkpoint therapy was first exploited with the success of anti-cytotoxic $\mathrm{T}$ lymphocyte antigen-4 (CTLA-4) in clinical treatment (2). Another immune checkpoint therapy includes antibodies against programmed death 1 (PD-1) or its ligand PD-L1 (3), and this immunotherapy has

Correspondence to: Dr Peihua Wang, Department of Ear, Nose and Throat, The Ninth Affliated Hospital of Shanghai Jiao Tong University, 639 Huangpu District Manufacturing Bureau Road, Shanghai 200011, P.R. China

E-mail: peihua_w@sohu.com

Key words: miR-375, PD-L1, JAK2, STAT3, HNSCC been confirmed effective for head and neck squamous cell carcinoma (HNSCC) treatment (4). However, most patients do not respond to anti-PD1 therapy or develop immunoresistance. Therefore, there is an urgent need to identify new modulators that can prevent immunoresistance or enhance immunotherapy. miRNAs are a type of 18-24 nucleotide non-coding RNAs with the ability to regulate messenger RNA (mRNA) expression via binding to 3 ' untranslated regions (UTRs) (5). Previous studies have indicated that miRNAs play critical roles in tumor proliferation, metastasis and many other processes (6). However, the roles and the related mechanisms of miRNAs in HNSCC immunotherapy have not been well elucidated.

Previous study has shown that interferon- $\gamma($ IFN- $\gamma)$ is a major $\mathrm{CD}^{+} \mathrm{T}$ cell effector cytokine and is prerequisite to constitutive PD-L1 expression (7). Thus, inhibition of IFN- $\gamma$ signaling downregulates PD-L1 expression and increases tumor lysis (8). IFN- $\gamma$ secretion from natural killer (NK) cells activates the JAK2/STAT1 pathway and further increases PD-L1 expression (8). Blockade of the JAK pathway could be a potential method to act synergistically with other immune therapies (9). Notably, bioinformatics assay indicated that JAK2 is a potential target of miR-375 which is downregulated in HNSCC. Additionally, miR-375 inhibits the metastasis of colorectal cancer via targeting SP1 (10). miR-375 was found to suppress metastasis by directly targeting SHOX2 in esophageal squamous cell carcinoma (11). Although it has been previously reported that JAK2 is a target of miR-375 in gastric cancer $(6,12)$, the roles of miR-375/JAK2 signaling in HNSCC immunotherapy remain unclear.

In the present study, we hypothesized that miR-375 inhibits STAT1-dependent PD-L1 expression induced by IFN- $\gamma$ exposure in HNSCC cells, and we assessed the roles of JAK2/STAT1 signaling in this process. We provide evidence that miR-375-mediated inhibition of IFN- $\gamma$-induced PD-L1 expression in HNSCC cells may allow T cells to create an antitumor immune environment.

\section{Materials and methods}

Cell culture and patient samples. HNSCC cell lines Hp-2 and FaDu were purchased from the Chinese Academy of Sciences Cell Bank. Jurkat cells, a human T leukemia cell line, were 
purchased from the American Type Culture Collection (ATCC; Manassas, VA, USA). HNSCC cell lines were maintained in Dulbecco's minimum essential medium (DMEM), and Jurkat T leukemia cells were maintained in RPMI-1640 medium, supplemented with $10 \%$ fetal bovine serum (FBS) (all from Gibco, Grand Island, NY, USA), $100 \mathrm{U} / \mathrm{ml}$ penicillin and $100 \mu \mathrm{g} / \mathrm{ml}$ streptomycin in a humidified atmosphere with $5 \% \mathrm{CO}_{2}$ at $37^{\circ} \mathrm{C}$. Paired mRNA profiling data were downloaded from the TCGA, The Cancer Genome Atlas (TCGA) data portal (http://cancergenome.nih.gov). The dataset from the Tumor Head Neck Squanmous Cell Carcinoma-TCGA520-rsem-tcgars (http://hgserver1.amc.nl/cgi-bin/r2/main. cgi), which includes 520 HNSCC samples, was obtained as a validation set. The $\mathrm{R} 2$ platform was used to analyze the microarray (http://r2.amc.nl).

Reagents. miR-375 mimics/inhibitor and the related negative control (NC) were synthesized by GenePharma, Inc. (Shanghai, China). Recombinant human IFN- $\gamma$ (cat. no. 300-02) was purchased from PeproTech (Rocky Hill, NJ, USA). Phycoerythrin (PE)-conjugated anti-human PD-L1 Ab (cat. no. 560795; dilution rate, 1:5,000) and PE-conjugated anti-human PD-1 Ab (cat. no. 561272; dilution rate, 1:5,000) were purchased from BD Biosciences (San Jose, CA, USA).

Cell viability assay. Cells with different treatment were seeded into 96 -well plates at $3 \times 10^{3}$ cells/well and incubated at $37^{\circ} \mathrm{C}$ for 24,48 and $72 \mathrm{~h}$, respectively. The cell viability was measured using a Cell Counting Kit-8 assay (cat. no. C0037; Beyotime, Nanjing, China) following the manufacturer's protocol.

Flow cytometric assay. Hep-2 and FaDu cells were seeded into 6-well plates at $2 \times 10^{5}$ or $4 \times 10^{5}$ cells/well overnight, respectively. Cells were added with $10 \mathrm{ng} / \mathrm{ml} \mathrm{IFN}-\gamma$, and then transfected with miR-375 mimics $(50 \mathrm{nM})$ and the related $\mathrm{NC}(50 \mathrm{nM})$ by Lipofectamine 2000 (Thermo Fisher Scientific, Inc., Waltham, MA, USA) for $48 \mathrm{~h}$. Then, the cells were washed with ice-cold PBS and re-suspended in flow cytometry buffer $\left(0.2 \% \mathrm{NaN}_{3}\right.$ and $1 \%$ BSA in PBS). Jurkat cells in fully supplemented RPMI1640 medium were also maintained as suspension cultures. Furthermore, the cells were stained with $0.5 \mu \mathrm{g}$ of the desired fluorophore-conjugated $\mathrm{Ab}$ or isotype control for $30 \mathrm{~min}$ in the dark at $4^{\circ} \mathrm{C}$, then washed twice with flow cytometry buffer and fixed in $1 \%$ paraformaldehyde. Fluorescence data were acquired using a FACSCalibur flow cytometer and BD CellQuest ${ }^{\mathrm{TM}}$ software (BD Biosciences). Counts/sample $\left(2 \times 10^{4}\right)$ were analyzed for all fluorescence experiments using FCS Express software (De Novo Software, Glendale, CA, USA).

$T$ cells and HNSCC cell co-cultures. After $24 \mathrm{~h}$ of transfection with miR-375 mimics ( $50 \mathrm{nM}$ ) using Lipofectamine 2000 and/or IFN- $\gamma(10 \mathrm{ng} / \mathrm{ml})$, HNSCC cells were collected, washed and re-suspended in fully supplemented DMEM, and further seeded into 24 -well plates at $2 \times 10^{5}$ or $40 \times 10^{5}$ cells/well for $6 \mathrm{~h}$ to allow attachment. Jurkat T leukemia cells stained with Oregon Green 488 dye were added to HNSCC cell monolayers at $5 \times 10^{4}$ cells/well. The resulting co-cultures were maintained for another $72 \mathrm{~h}$. Then, the supernatants were centrifuged and collected for examination of IL-2. The proliferation of Jurkat $\mathrm{T}$ cells was analyzed by flow cytometry. The number of cell divisions was calculated using the formula: Mean fluorescence intensity (MFI) control $=2^{\mathrm{n}} \times \mathrm{MFI}_{\text {sample }}$, where $M \mathrm{I}_{\text {control }}$ is the MFI of the non-proliferative control and $n$ is the number of cell divisions (9). Cell divisions were normalized to the medium control.

Cytokine measurement. IL-2 level was measured in cell supernatants from T cells and HNSCC cell co-cultures using IL-2-specific ELISA kit from Boster (EK0397; Wuhan, China) according to the manufacturer's instructions.

RNA immune co-precipitation (RIP) assay. The procedure for RIP assay was performed as previously described (13). RNA in the IP materials was measured by qRT-PCR.

Plasmid construction and transfection. JAK2 coding sequences were inserted into the pcDNA3.1 (+) plasmid, named as JAK2-CDS. Cells were seeded into 6-well plates at a density of $2.5 \times 10^{5}$ cells/well. Fifty nanomoles of miRNA mimics or NC, and JAK2-CDS were transfected into the cells using Lipofectamine 2000 reagent according to the manufacturer's instructions.

Quantitative real-time PCR ( $q R T$-PCR). Total RNA was extracted from cells using TRIzol reagent (Invitrogen, Carlsbad, CA, USA) according to the manufacturer's instructions. cDNA was reverse transcribed with M-MLV transcriptase (Promega, Madison, WI, USA). qRT-PCR was performed on the ABI StepOnePlus Real-Time PCR system [Applied Biosystems (ABI,) Foster City, CA, USA USA] using SYBR-Green PCR kit (Takara, Japan). U6 and GAPDH were used as the internal controls for miRNA and mRNA expression, respectively. The primers of JAK2 for qRT-PCR are listed below: forward, 5'-CTGCAGGAAGGAGAGAGGAAGAGGA-3' and reverse, 5'-GAATGTTATTGGCAGTCAG-3'. Relative quantification was calculated using the $2^{-\Delta \Delta \mathrm{Ct}}$ method.

Western blotting. Cells were lysed with radioimmunoprecipitation assay (RIPA) lysis buffer containing protease and phosphatase inhibitors. The concentration of protein was quantified by BCA (bicinchoninic acid) protein assay kit (Beyotime). Equal amount of protein was loaded onto $10 \%$ SDS-PAGE, followed by wet transferring to polyvinylidene fluoride (PVDF) membranes. The membranes were blocked with 5\% non-fat milk at room temperature for $1 \mathrm{~h}$, and then incubated with primary antibodies at $4^{\circ} \mathrm{C}$ overnight. The membranes were subsequently blotted with HRP-conjugated secondary antibodies and developed with the ECL detection system (Thermo Fisher Scientific, Inc.).

Luciferase reporter analysis. For the luciferase assay, the full length of JAK2 3'UTR containing the wild-type or the mutant binding sites for miRNA-375 was cloned into the pMIR-Report vector downstream of the luciferase reporter gene, which was denoted as Luc-JAK2-3'UTR-WT and Luc-JAK2-3'UTR-MUT, respectively. These constructs were co-transfected with miR-375 mimics or NC in HNSCC cells using Lipofectamine 2000. Luminescent signals were measured $48 \mathrm{~h}$ later by luminometer (Thermo Fisher Scientific, Inc.). $\beta$-gal was used to normalize the luciferase activity. 
A
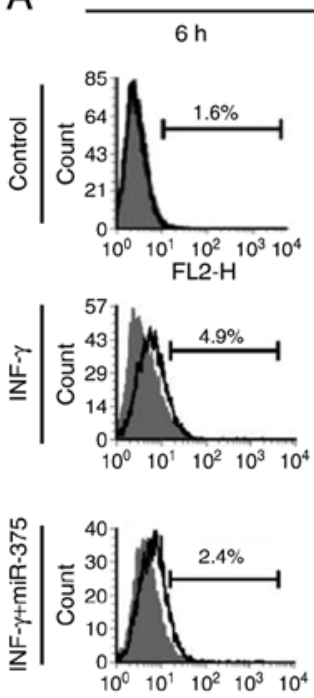

C

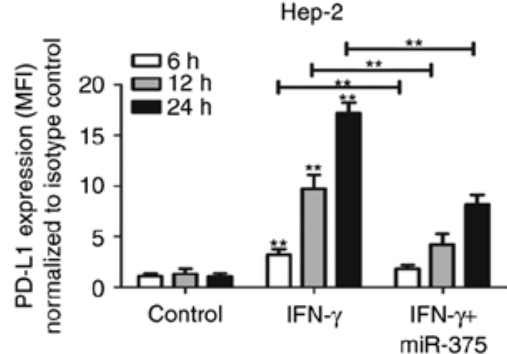

B
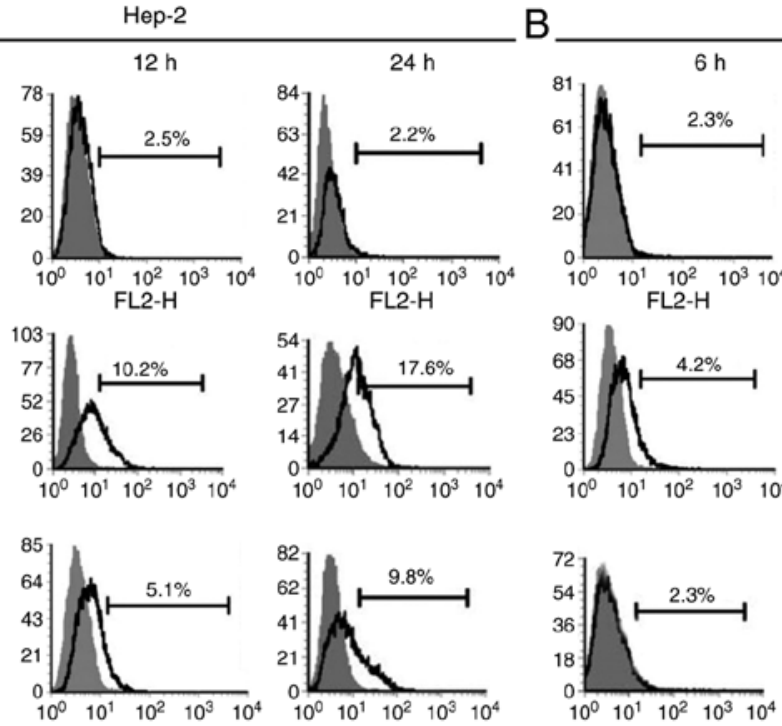

D

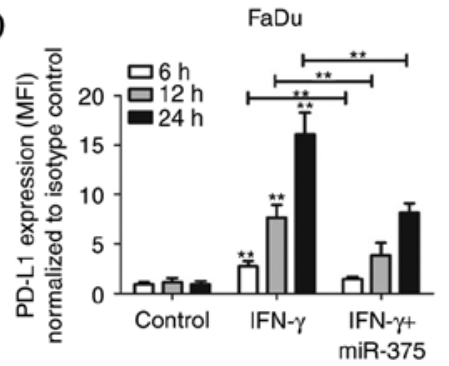

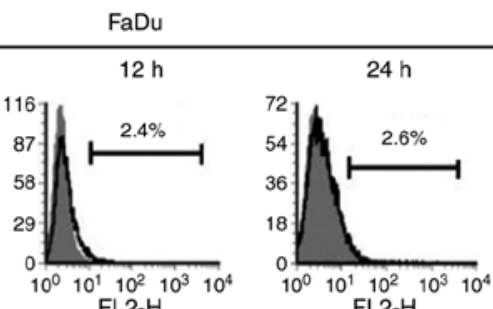
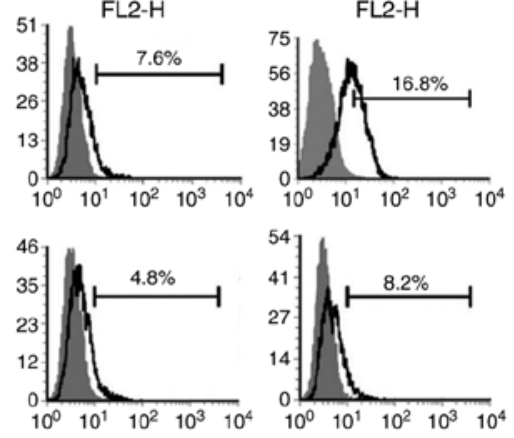

E

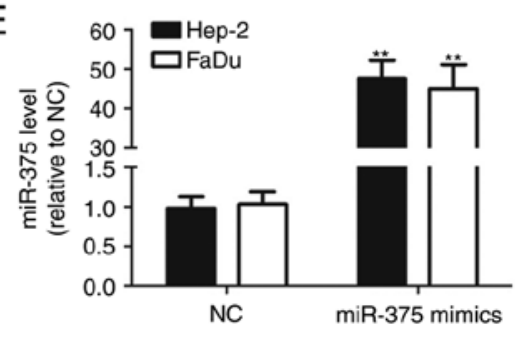

Figure 1. miR-375 inhibits IFN- $\gamma$-induced PD-L1 expression in HNSCC cells. (A-D) The average mean fluorescence intensity (MFI) of PD-L1 from 3 independent experiments \pm SD. (E) The transfection efficiency of miR-375 was examined in Hep-2 and FaDu cells via qRT-PCR assay. Flow cytometry values have been normalized by subtracting the appropriate isotype control value; ${ }^{* *} \mathrm{P}<0.01$. PD-L1, programmed death 1 ligand 1 ; HNSCC, head and neck squamous cell carcinoma.
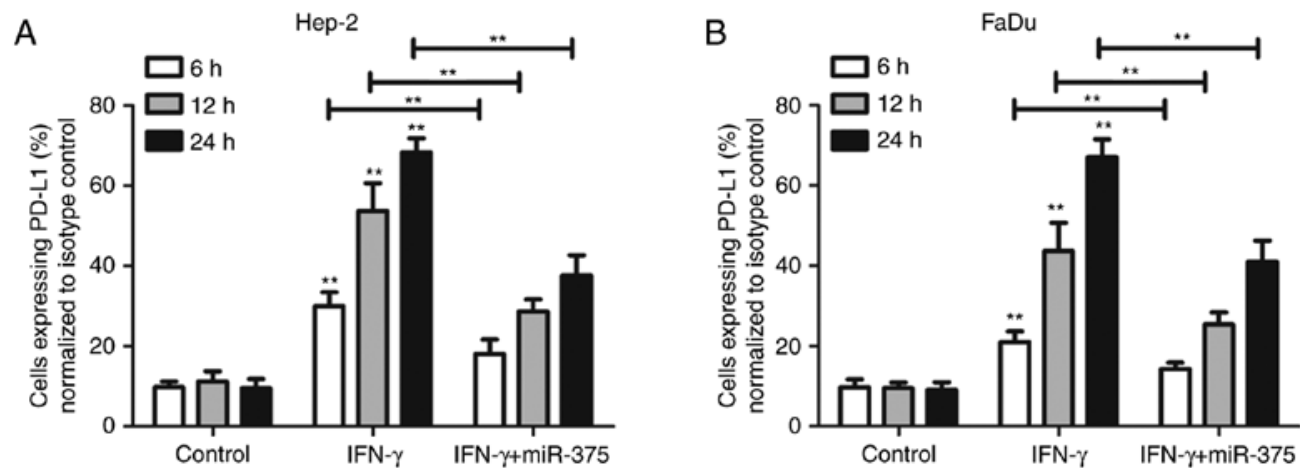

Figure 2. miR-375 reduces the IFN- $\gamma$-induced percentage of PD-L1-expressing HNSCC cells. (A and B) The average percentage of cells expressing PD-L1 from 3 independent experiments \pm SD. Flow cytometry values have been normalized by subtracting the appropriate isotype control value; ${ }^{* *} \mathrm{P}<0.01$. PD-L1, programmed death 1 ligand 1; HNSCC, head and neck squamous cell carcinoma.

Statistical analysis. Data are presented as the mean \pm standard deviation (SD). The differences between the groups were analyzed using ANOVA with the Tukey-Kramer post test, and $\mathrm{P}<0.05$ was considered to indicate a statistically significant result.

\section{Results}

miR-375 inhibits IFN- $\gamma$-induced PD-L1 expression in HNSCC cells. Consistent with previous studies $(7,8)$, we found that
IFN- $\gamma$-treated Hep-2 and FaDu cells showed a time-dependent increase in PD-L1 expression (Fig. 1A-D). However, this effect was attenuated following co-transfection with miR-375. The transfection efficiency of miR-375 mimics was confirmed by qRT-PCR, and the result indicated that transfection with miR-375 mimics significantly upregulated miR-375 level in Hep-2 and FaDu cells (Fig. 1E). Notably, miR-375 reduced both the percentage of PD-L1-expressing HNSCC cells and the amount of PD-L1 expressed in individual HNSCC cells (Fig. 2A and B). 
A

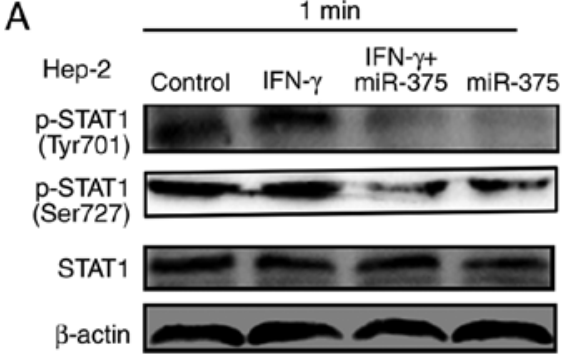

$\mathrm{B}$
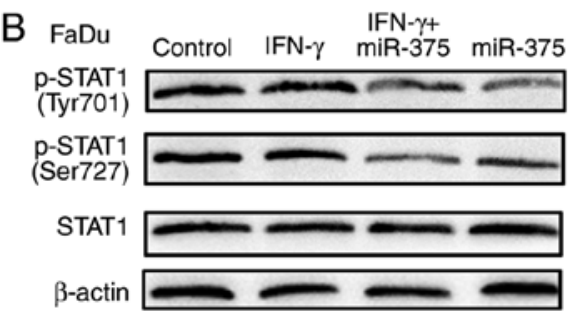

$10 \mathrm{~min}$
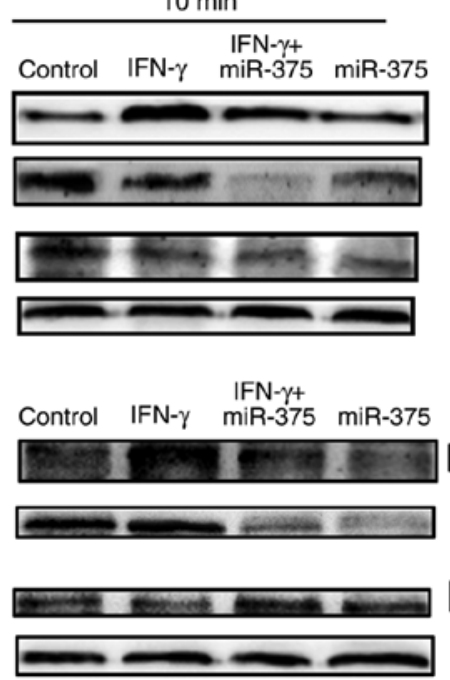

$30 \mathrm{~min}$

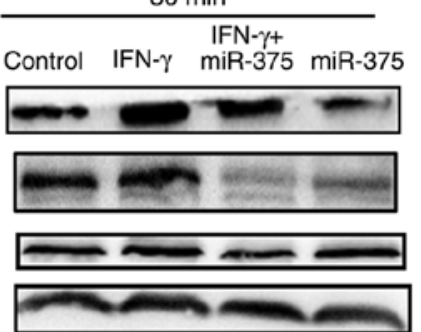

IFN- $\gamma+$

Control IFN- $\gamma$ miR-375 miR-375
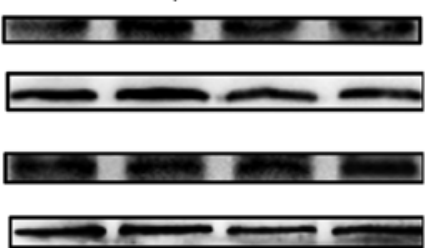

Figure 3. miR-375 inhibits IFN- $\gamma$-induced phosphorylation of STAT1 in HNSCC cells. (A) Hep-2 and (B) FaDu cells were pre-transfected with miR-375 mimics, and then incubated in the absence or presence of $10 \mathrm{ng} / \mathrm{ml} \mathrm{IFN-} \gamma$ for 1,10 or $30 \mathrm{~min}$. Cell lysates were collected and STAT1 phosphorylation at Tyr701 and Ser727 was determined by western blotting. Data shown are a representative western blotting from each time point and the average level of phosphorylated STAT1 (Tyr701 and Ser727) normalized to total STAT1 from 3 independent experiments. HNSCC, head and neck squamous cell carcinoma.

A

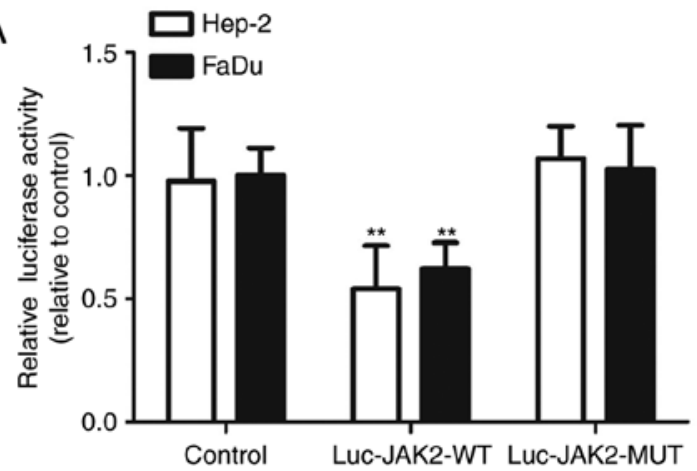

C

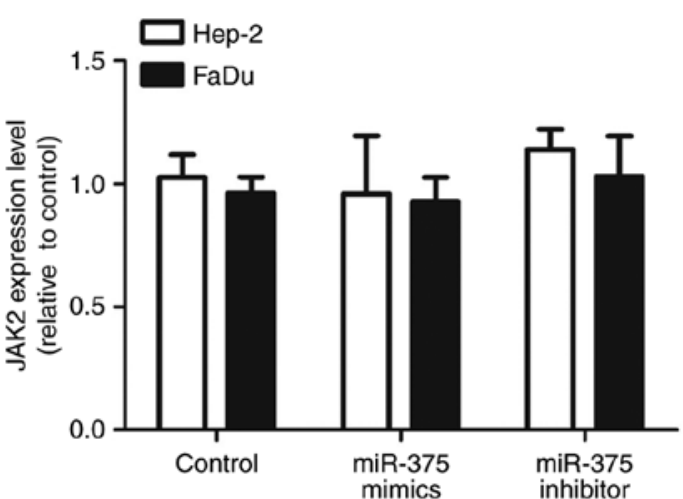

B

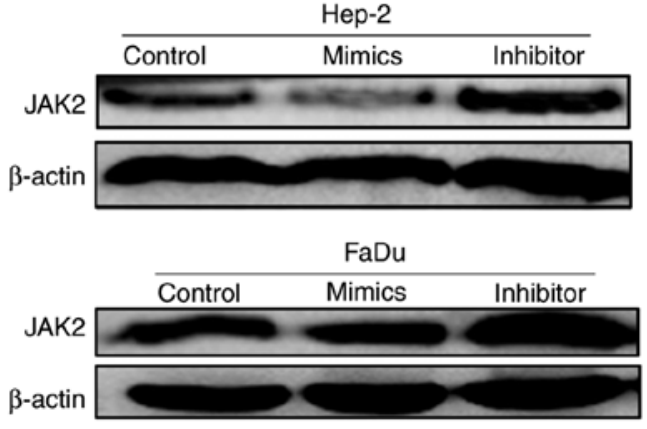

D

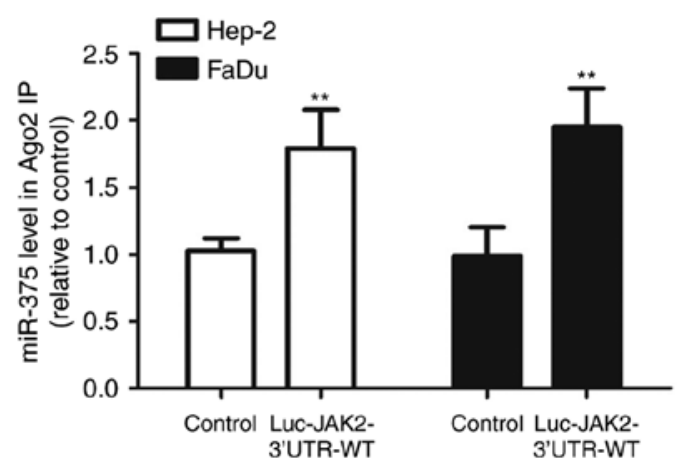

Figure 4. JAK2 is a bona fide target of miR-375 in HNSCC cells. (A) HNSCC cells were co-transfected with recombinant Luc-JAK2-3'UTR-WT and LucJAK2-3'UTR-MUT, miR-375 mimics or NC, and pMIR-Report $\beta$-gal control plasmid for $72 \mathrm{~h}$, then the luciferase activity was measured and normalized to $\beta$-gal activity. qRT-PCR (B) and western blotting (C) analyses of JAK2 expression in Hep-2 and FaDu cells after transfection of miR-375 mimics and inhibitor for $72 \mathrm{~h} ; \beta$-actin expression was used as an internal control. (D) RIP assay was performed using an anti-Ago 2 antibody or control IgG and lysates from HNSCC cells transfected with JAK2 3'UTR and control, and then miR-375 level was measured by qRT-PCR; ${ }^{* *}$ P<0.01. HNSCC, head and neck squamous cell carcinoma.

miR-375 inhibits IFN- $\gamma$-induced STATl activation. Previous research has shown that IFN- $\gamma$ induction activates JAK1 and JAK2, further leading to the phosphorylation of STAT1 (8). We found this to be the case in HNSCC cells. Treatment of HNSCC cells with $10 \mathrm{ng} / \mathrm{ml}$ IFN- $\gamma$ caused STAT1 phosphorylation (Fig. 3) at different time points. Additionally, transfection with miR-375 mimics before IFN- $\gamma$ treatment decreased the STAT1 phosphorylation level (Fig. 3), indicating that ablation of STAT1 phosphorylation is necessary for the inhibitory effects of miR-375 on IFN- $\gamma$-induced PD-L1 expression. 

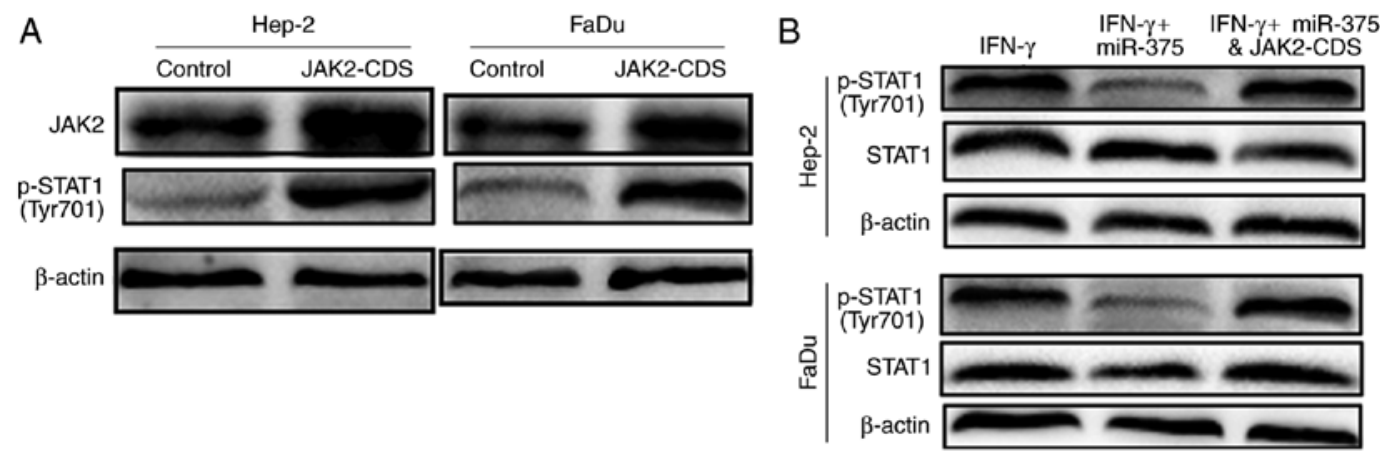

Figure 5. miR-375 inhibits IFN- $\gamma$-induced p-STAT1 activation in a JAK2-dependent manner. (A) The expression levels of JAK2 and p-STAT1 (Tyr701) were detected in HNSCC cell with or without JAK2-CDS introduction. (B) Hep-2 and FaDu cells were pre-transfected with miR-375 mimics plus JAK2-CDS or not for $48 \mathrm{~h}$, and then treated with IFN- $\gamma$. The expression level of p-STAT1 (Tyr701) was examined.

A

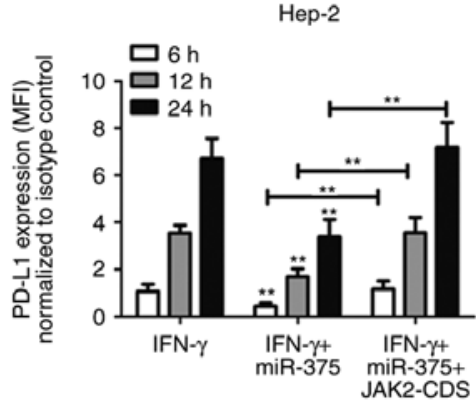

D

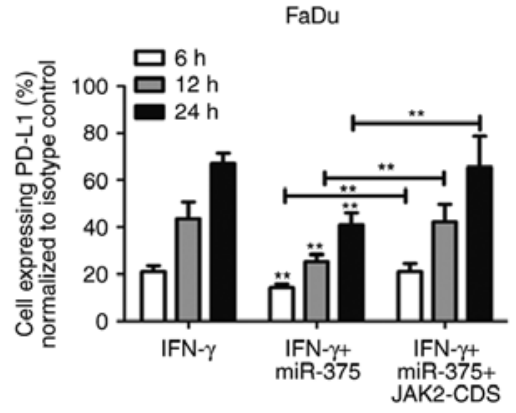

G

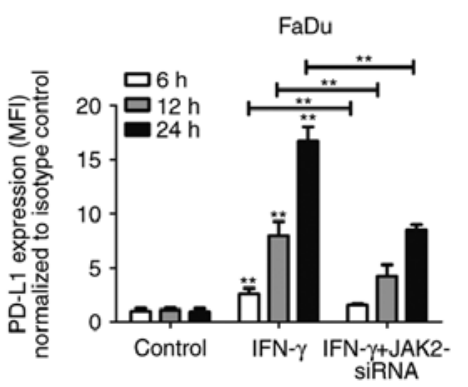

B

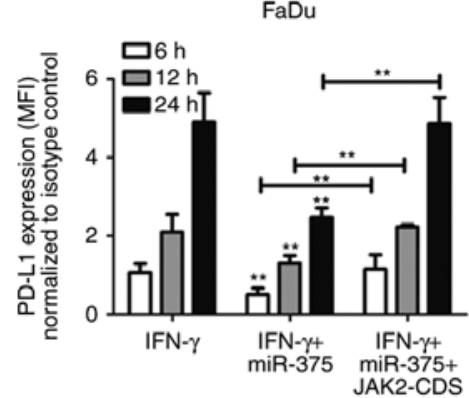

E

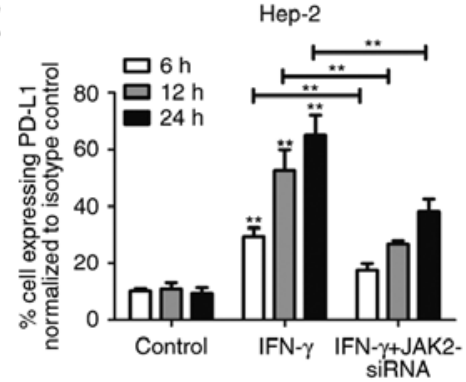

$\mathrm{H}$

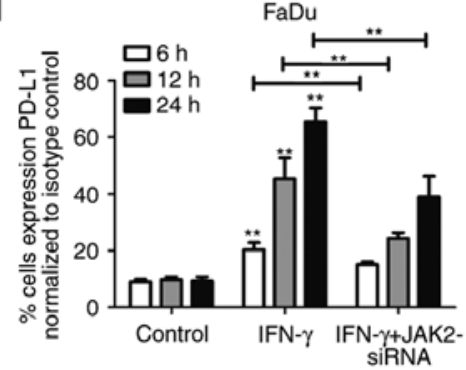

C

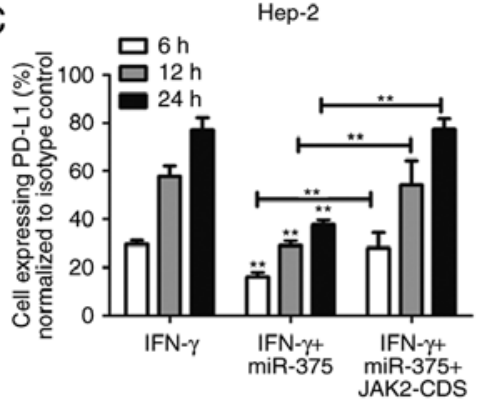

$\mathrm{F}$

Hep-2

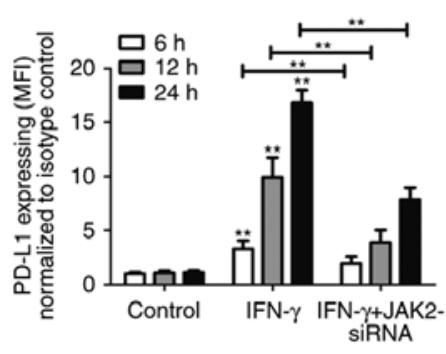

I

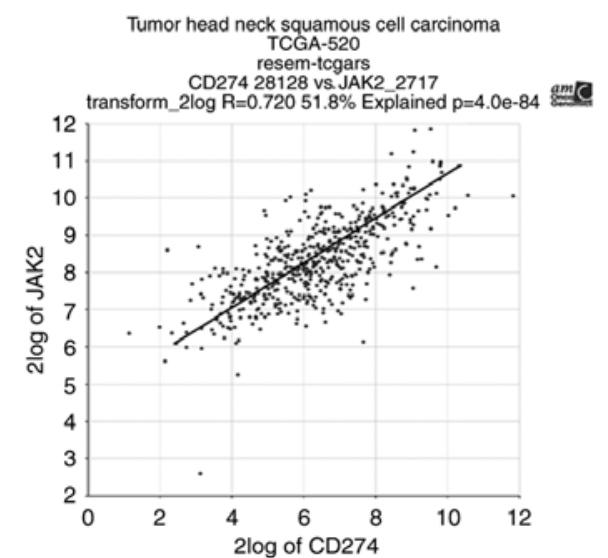

Figure 6. miR-375 inhibits IFN- $\gamma$-induced PD-L1 expression in a JAK2-dependent manner. (A and B) The average mean fluorescence intensity (MFI) of PD-L1 from 3 independent experiments \pm SD in cells depicted in (Fig. 5B). (C and D) The average percentage of cells expressing PD-L1 from 3 independent experiments \pm SD in cells depicted in (Fig. 5B). (E-H) (E and H) The average percentage of cells expressing PD-L1 from 3 independent experiments \pm SD (F and G) The average mean fluorescence intensity (MFI) of IFN $\gamma$ receptor from 3 independent experiments \pm SD and the average percentage of cells expressing IFN $-\gamma$ receptor from 3 independent experiments \pm SD. Flow cytometry values have been normalized by subtracting the appropriate isotype control value. (I) The expression of JAK2 and PD-L1 in HNSCC tissues exhibited a positive correlation ( $\mathrm{P}=4.0 \mathrm{e}-84) ;{ }^{* *} \mathrm{P}<0.01$. PD-L1, programmed death 1 ligand 1; HNSCC, head and neck squamous cell carcinoma. 
A
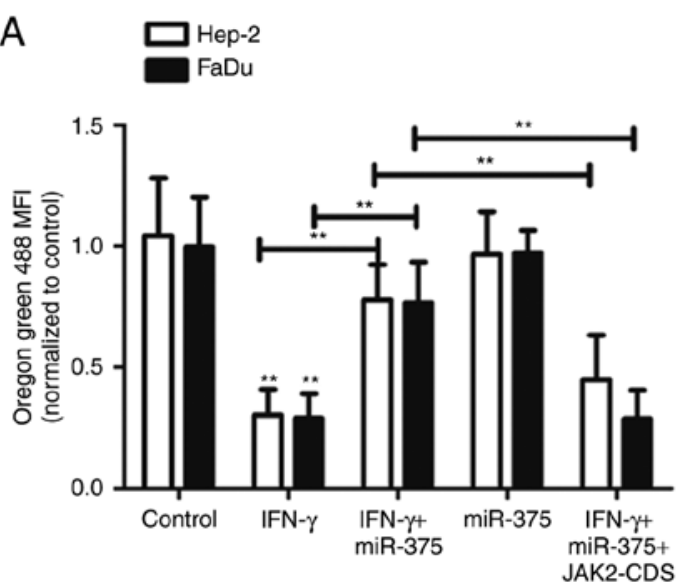

B

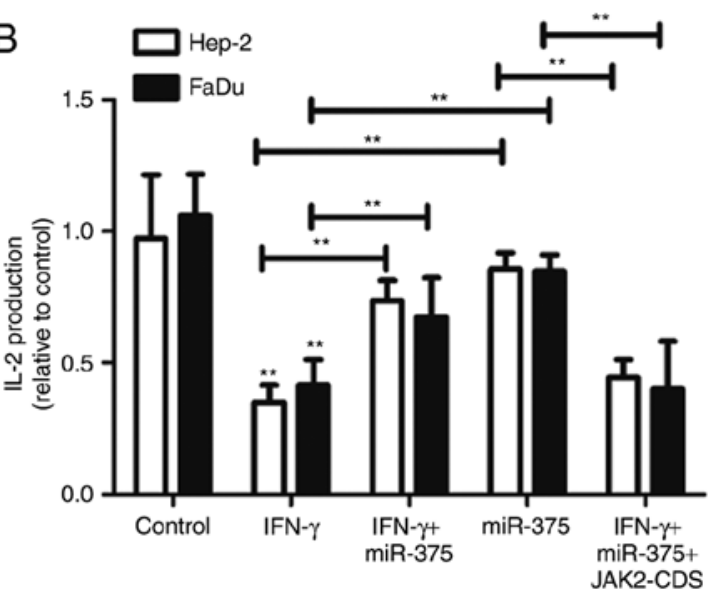

Figure 7. Upregulation of miR-375 contributes to increased T cell proliferation in HNSCC co-cultures. (A) HNSCC cells were pre-transfected with miR-375 mimics plus JAK2-CDS or not and then treated with $10 \mathrm{ng} / \mathrm{ml} \mathrm{IFN-} \gamma$, and co-cultured with Oregon Green 488 -stained Jurkat T cells. After $72 \mathrm{~h}$, Jurkat T cells were collected and the level of fluorescence was determined by flow cytometry. The average mean fluorescence intensity (MFI) of each sample normalized to the average MFI of the medium control from 3 independent experiments \pm SD. (B) IL-2 release was tested by ELISA in cells depicted in (A) for $48 \mathrm{~h}$; ${ }^{* *} \mathrm{P}<0.01$. HNSCC, head and neck squamous cell carcinoma.

A

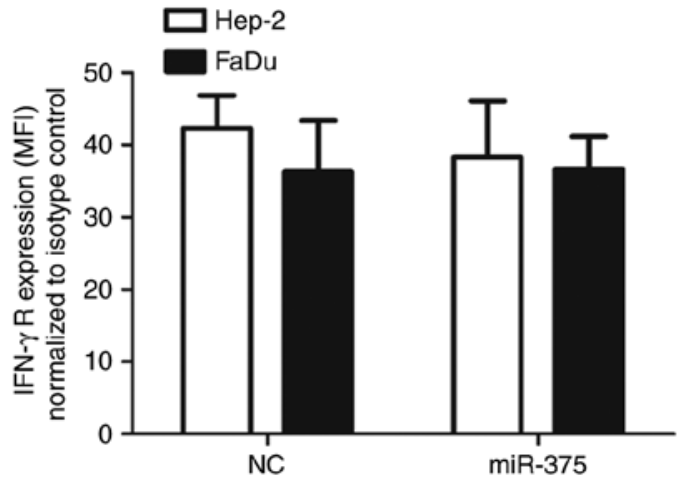

B

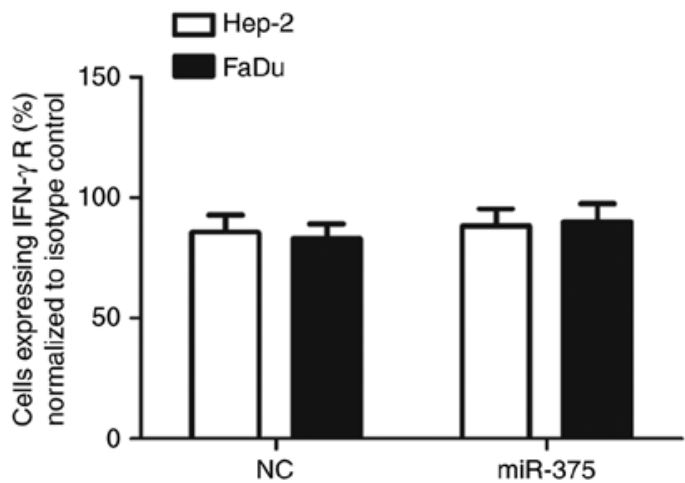

Figure 8. Expression of IFN- $\gamma$ receptor on HNSCC cells was not altered following the transfection of miR-375. (A and B) The average mean fluorescence intensity (MFI) of IFN $-\gamma \mathrm{R}$ from 3 independent experiments \pm SD and the average percentage of cells expressing IFN- $\gamma \mathrm{R}$ - from 3 independent experiments \pm SD Flow cytometry values have been normalized by subtracting the appropriate isotype control value; ${ }^{* *} \mathrm{P}<0.01$. HNSCC, head and neck squamous cell carcinoma.

HNSCC cells. As shown in Fig. 4A, co-transfection with miR-375 mimics and the Luc-JAK2-3'UTR-WT construct significantly reduced the luciferase activity; whereas the luciferase activity of Luc-JAK2-3'UTR-MUT was unaffected by miR-375 transfection. Additionally, western blot results showed that upregulation of miR-375 markedly decreased JAK2 protein expression in Hep-2 and FaDu cells, while transfection with miR-375 inhibitor increased JAK2 protein expression (Fig. 4B). However, no significant difference was observed at the JAK2 mRNA level (Fig. 4C), indicating that miR-375 regulates JAK2 expression at the post-transcriptional level. To further confirm the direct binding between miR-375 and JAK2 3'UTR in HNSCC cells, JAK2 3'UTR was introduced into HNSCC cells by Luc-JAK2-3'UTR-WT transfection, and RIP assay was performed to detect the bound miRNAs in the Ago2-binding complex. As shown in Fig. 4D, the miR-375 level which bound to Ago2 was increased in the Luc-JAK23'UTR-WT-introduced cells. Consequently, we demonstrated that JAK2 is a direct target of miR-375 in HNSCC cells.

miR-375 inhibits IFN- $\gamma$-induced STAT1 activation in a JAK2dependent manner. To further confirm the essential role of
JAK2 in miR-375-mediated inhibition on IFN- $\gamma$-induced STAT1 activation. JAK2 was overexpressed in Hep-2 and FaDu cells by transfection with JAK2-CDS. The transfection efficiency of JAK-CDS was confirmed by western blot analysis (Fig. 5A). Overexpression of JAK2 significantly upregulated the phosphorylation level of STAT1. Additionally, co-transfection with JAK2-CDS and miR-375 reversed the inhibitory effects of miR-375 on IFN- $\gamma$-induced STAT1 activation (Fig. 5B) and PD-L1 expression (Fig. 6A-D). Moreover, knockdown of JAK2 also reduced IFN- $\gamma$-induced PD-L1 expression (Fig. 6E-H). Importantly, by analyzing the mRNA expression in the mRNA microarrays from TCGA, we confirmed that the levels of JAK2 and PD-L1 were positively correlated $(\mathrm{P}=4.0 \mathrm{e}-84)$ in HNSCC patients (Fig. 6I). All together, these results indicate that JAK2/STAT1 signaling by miR-375 participates in IFN- $\gamma$-induced STAT1 activation and thus suppresses PD-L1 expression.

Upregulation of miR-375 contributes to increased $T$ cell proliferation in HNSCC co-cultures. Having shown that miR-375 inhibited IFN- $\gamma$-induced STAT1 activation and thus decreased PD-L1 expression, we sought to investigate the 
potential relevance of this phenotype with $\mathrm{T}$ cell proliferation. We co-cultured Oregon Green 488-stained Jurkat T cells with Hep-2 and FaDu cells that were previously treated

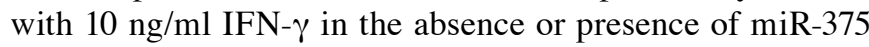
overexpression. After $72 \mathrm{~h}$ of co-culture, $\mathrm{T}$ cell proliferation was tested by flow cytometry. Fig. 7A showed that $\mathrm{T}$ cell proliferation, characterized as decreased MFI, was reduced in the presence of IFN- $\gamma$-treated Hep- 2 and FaDu cells, and this effect was presumably caused by the inhibition of PD-1/PD-L1 interaction. Most importantly, T cell proliferation was reversed to the control level in co-cultures of Hep-2 and FaDu cells with miR-375 introduction after IFN- $\gamma$ treatment. In addition, introduction of JAK2-CDS in HNSCC cells reversed the miR-375-mediated effects. Furthermore, IL-2 concentration secreted by Jurkat $\mathrm{T}$ cells was upregulated in the co-cultures when HNSCC cells were treated with miR-375 plus IFN- $\gamma$ compared with the co-cultures of HNSCC cells with IFN- $\gamma$ treatment only (Fig. 7B); this result was reversed by JAK 2 overexpression. Collectively, our findings demonstrate that the inhibitory effects of miR-375 on IFN- $\gamma$-induced expression of PD-L1 in HNSCC cells is associated with increased $\mathrm{T}$ cell responses in co-cultures and dependent on JAK2 expression.

\section{Discussion}

Low-level expression of miR-375 has been shown to be correlated with poor outcome and metastasis while altering the invasive properties of head and neck squamous cell carcinomas (14). Additionally, miRNA-375 suppresses extracellular matrix degradation and invadopodial activity via regulating oncogene AEG-1/MTDH in HNSCC $(15,16)$. However, the immune regulatory roles and the related mechanisms of miR-375 in HNSCC have not been defined, and the mechanisms that underlie IFN- $\gamma$-induced expression of PD-L1 have not been clearly elucidated.

miR-375 has been extensively explored for its inhibitory effects on tumor development $(6,10,11)$. However, to the best of our knowledge, the present study is the first to provide evidence of a potential role of miR-375 in improving a $\mathrm{T}$ cell-mediated antitumor immune response against HNSCC cells by modulating IFN- $\gamma$-induced expression of PD-L1, which is validated as a promising target for immunotherapy in HNSCC treatment $(4,17)$. We indicated here that miR-375 inhibited IFN- $\gamma$-induced PD-L1 expression via targeting JAK2 and thus inactivating JAK2/STAT1 signaling This means that miR-375 inhibits PD-1/PD-L1-mediated immune escape in HNSCC patients whose tumor-associated PD-L1 expression is elevated due to IFN- $\gamma$ inductive effects or JAK2/STAT1mediated pro-inflammatory cytokines, such as IL-2 in the tumor microenvironment.

Specifically, although JAK2 phosphorylated STAT1 at Tyr701 and Ser727 residues, phosphorylation of STAT1 at the Tyr701 residue is independent of Ser727 phosphorylation (18). In addition, the rapid phosphorylation of STAT1 (pY701), but not activation of other STATs is the main mediator of IFN- $\gamma$ induction and suppresses tumor cell susceptibility to NK cells through upregulation of PD-L1 (8). Our results showed that treating HNSCC cells with miR-375 caused a significant and early decrease in IFN- $\gamma$-induced phosphorylation of STAT1 at Tyr701. However, miR-375 still could decrease the phosphorylation level of STAT1 (pS727), and this could be due to miR-375-mediated inactivation of JAK2 which could phosphorylate STAT1 at Tyr701 and Ser727 residues. More importantly, the inhibitory effect of miR-375 on STAT1 phosphorylation was not due to the decreased expression of the IFN- $\gamma$ receptor on HNSCC cells (Fig. 8A and B). Rather, since our results indicate that miR-375 directly targets JAK2, it follows that miR-375 can affect JAK2-dependent PD-L1 expression induced by IFN- $\gamma$ treatment of HNSCC cells. As PD-L1 upregulation is a common immune escape of tumor cells (19), and higher PD-L1 expression in tumor patients predicates a poor prognosis in various cancers, including HNSCC (20). Previous study has shown that PD-L1/PD-1 interaction contributes to the functional suppression of T-cell responses characterized as impaired IL-2 production by Jurkat $T$ cells (21). Indeed, our results showed that the inhibitory effect of miR-375 on IFN- $\gamma$-induced PD-L1 expression by HNSCC cells was associated with increased proliferation and IL-2 synthesis by Jurkat T cells that were co-cultured with HNSCC cells, which is consistent with an attenuation of PD-1/PD-L1 interactions. Future studies could be performed to confirm this immune-modulating effects of miR-375 in vivo using immune-competent mice. Additionally, since miR-375 could target JAK2 in gastric tumor, the effects of miR-375 on IFN- $\gamma$-induced PD-L1 expression should also be explored. Furthermore, previous research has shown that miR-375 suppresses the invasive properties of HNSCC (14). miR-375 therefore has the potential to modulate HNSCC growth in an indirect or direct manner, indicating an important potential therapeutic target and providing a strong rationale for the development of miRNA-based therapeutic strategies for HNSCC.

\section{References}

1. Khalil DN, Smith EL, Brentjens RJ and Wolchok JD: The future of cancer treatment: Immunomodulation, CARs and combination immunotherapy. Nat Rev Clin Oncol 13: 394, 2016.

2. Sharma P, Wagner K, Wolchok JD and Allison JP: Novel cancer immunotherapy agents with survival benefit: Recent successes and next steps. Nat Rev Cancer 11: 805-812, 2011.

3. Ansell SM, Lesokhin AM, Borrello I, Halwani A, Scott EC, Gutierrez M, Schuster SJ, Millenson MM, Cattry D, Freeman GJ, et al: PD-1 blockade with nivolumab in relapsed or refractory Hodgkin's lymphoma. N Engl J Med 372: 311-319, 2015.

4. Saâda-Bouzid E, Defaucheux C, Karabajakian A, Coloma VP, Servois V, Paoletti X, Even C, Fayette J, Guigay J, Loirat D, et al: Hyperprogression during anti-PD-1/PD-L1 therapy in patients with recurrent and/or metastatic head and neck squamous cell carcinoma. Ann Oncol 28: 1605-1611, 2017.

5. Li X, Zheng L, Zhang F, Hu J, Chou J, Liu Y, Xing Y and Xi T: STARD13-correlated ceRNA network inhibits EMT and metastasis of breast cancer. Oncotarget 7: 23197-23211, 2016.

6. Miao L, Liu K, Xie M, Xing Y and Xi T: miR-375 inhibits Helicobacter pylori-induced gastric carcinogenesis by blocking JAK2-STAT3 signaling. Cancer Immunology Immunother 63: 699-711, 2014.

7. Lee SJ, Jang BC, Lee SW, Yang YI, Suh SI, Park YM, Oh S, Shin JG, Yao S, Chen L and Choi IH: Interferon regulatory factor-1 is prerequisite to the constitutive expression and IFN-gamma-induced upregulation of B7-H1 (CD274). FEBS Lett 580: 755-762, 2006.

8. Bellucci R, Martin A, Bommarito D, Wang K, Hansen SH, Freeman GJ and Ritz J: Interferon- $\gamma$-induced activation of JAK1 and JAK2 suppresses tumor cell susceptibility to NK cells through upregulation of PD-L1 expression. Oncoimmunology 4: e1008824, 2015. 
9. Coombs MR, Harrison ME and Hoskin DW: Apigenin inhibits the inducible expression of programmed death ligand 1 by human and mouse mammary carcinoma cells. Cancer Lett 380: 424-433, 2016.

10. Cui F, Wang S, Lao I, Zhou C, Kong H, Bayaxi N, Li J, Chen Q, Zhu T and Zhu H: miR-375 inhibits the invasion and metastasis of colorectal cancer via targeting SP1 and regulating EMT-associated genes. Oncol Rep 36: 487-93, 2016

11. Yi J, Jin L, Chen J, Feng B, He Z, Chen L and Song H: MiR-375 suppresses invasion and metastasis by direct targeting of SHOX2 in esophageal squamous cell carcinoma. Acta Biochim Biophys Sin 49: 159-169, 2017.

12. Ding L, Xu Y, Zhang W, Deng Y, Si M, Du Y, Yao H, Liu X, Ke Y, Si J and Zhou T: MiR-375 frequently downregulated in gastric cancer inhibits cell proliferation by targeting JAK2. Cell Res 20: 784-793, 2010.

13. Sheng B, Zhao L, Zang X, Zhen J and Chen W: miR-375 ameliorates sepsis by downregulating miR-21 level via inhibiting JAK2-STAT3 signaling. Biomed Pharmacother 86: 254-261, 2017.

14. Harris T, Jimenez L, Kawachi N, Fan JB, Chen J, Belbin T, Ramnauth A, Loudig O, Keller CE, Smith R, et al: Low-level expression of miR-375 correlates with poor outcome and metastasis while altering the invasive properties of head and neck squamous cell carcinomas. Am J Pathol 180: 917-928, 2012.

15. Nohata N, Hanazawa T, Kikkawa N, Mutallip M, Sakurai D, Fujimura L, Kawakami K, Chiyomaru T, Yoshino H, Enokida $\mathrm{H}$, et al: Tumor suppressive microRNA-375 regulates oncogene AEG-1/MTDH in head and neck squamous cell carcinoma (HNSCC). J Hum Genet 56: 595-601, 2011.
16. Jimenez L, Sharma VP, Condeelis J, Harris T, Ow TJ, Prystowsky MB, Childs G and Segall JE: MicroRNA-375 suppresses extracellular matrix degradation and invadopodial activity in head and neck squamous cell carcinoma. Arch Pathol Lab Med 139: 1349-1361, 2015.

17. Ock CY, Kim S, Keam B, Kim M, Kim TM, Kim JH, Jeon YK, Lee JS, Kwon SK, Hah JH, et al: PD-L1 expression is associated with epithelial-mesenchymal transition in head and neck squamous cell carcinoma. Oncotarget 7: 15901-15914, 2016.

18. Zhu X, Wen Z, Xu LZ and Darnell JE Jr: Stat1 serine phosphorylation occurs independently of tyrosine phosphorylation and requires an activated Jak2 kinase. Mol Cell Biol 17: 6618-6623, 1997.

19. Pardoll DM: The blockade of immune checkpoints in cancer immunotherapy. Nat Rev Cancer 12: 252-264, 2012.

20. Mann JE, Hoesli R, Michmerhuizen NL, Devenport SN, Ludwig ML, Vandenberg TR, Matovina C, Jawad N, Mierzwa M, Shuman AG, et al: Surveilling the potential for precision medicine-driven PD-1/PD-L1-targeted therapy in HNSCC. J Cancer 8: 332-344, 2017.

21. Yang W, Chen PW, Li H, Alizadeh H and Niederkorn JY: PD-L1: PD-1 interaction contributes to the functional suppression of T-cell responses to human uveal melanoma cells in vitro. Invest Ophthalmol Vis Sci 49: 2518-2525, 2008. 